\title{
Solution Structure and Thermodynamics of 2',5' RNA Intercalation
}

\author{
Eric D. Horowitz ${ }^{1}$, Seth Lilavivat ${ }^{1}$, Benjamin W. Holladay ${ }^{1}$, \\ Markus W. Germann ${ }^{2}$, and Nicholas V. Hud ${ }^{1}$ \\ ${ }^{1}$ Parker H. Petit Institute of Bioengineering and Bioscience, Georgia Institute of \\ Technology, School of Chemistry and Biochemistry, Atlanta, GA, USA 30332-0400 \\ ${ }^{2}$ Georgia State University, Departments of Chemistry and Biology, Atlanta, GA, USA \\ 30302-4098
}

\section{Supporting Information}

\begin{tabular}{|c|c|c|c|c|c|c|c|c|}
\hline \multicolumn{9}{|c|}{ 2',5' RNA ${ }^{1} \mathrm{H}$ Assignments } \\
\hline & H6/H8 & H5 & H1' & H2' & H3' & H4' & H5'/5" & imino \\
\hline G1 & 7.89 & na & 5.89 & 4.98 & 4.42 & 4.15 & $3.70 / 2.75$ & 11.87 \\
\hline $\mathrm{C} 2$ & 7.44 & 5.67 & 5.72 & 4.15 & 4.16 & 3.49 & $3.91 / 3.85$ & na \\
\hline C3 & 7.48 & 5.48 & 6.02 & 4.70 & 4.47 & 4.25 & 4.05 & na \\
\hline G4 & 7.45 & na & 5.68 & 4.64 & 4.72 & 4.19 & -- & 11.30 \\
\hline C5 & 7.09 & 4.70 & 5.88 & 4.48 & 4.45 & 4.30 & 4.11 & na \\
\hline G6 & 7.39 & na & 5.71 & 4.70 & 4.18 & 4.16 & -- & 11.22 \\
\hline G7 & 7.11 & na & 5.73 & 4.48 & 4.16 & 4.27 & 4.14 & 12.72 \\
\hline C8 & 7.20 & 5.20 & 5.56 & 3.90 & 3.85 & 3.99 & $4.08 / 3.87$ & na \\
\hline
\end{tabular}

\section{2',5' RNA ${ }^{31} \mathrm{P}$ Assignments}

\begin{tabular}{|c|c|c|c|c|c|c|c|c|}
\hline G1pC2 & C2pC3 & C3pG4 & \multicolumn{2}{|c|}{ G4pC5 } & \multicolumn{2}{|c|}{ C5pG6 } & G6pG7 & \multirow{2}{*}{$\frac{\mathbf{G 7 p C 8}}{-0.57(0.15)}$} \\
\hline$-0.81(-0.86)$ & $-0.25(-0.10)$ & $0.87(-0$. & -1.23 & 0.61) & & $-0.16)$ & $-0.51(-0.75)$ & \\
\hline \multicolumn{9}{|c|}{ Proflavine ${ }^{1} \mathrm{H}$ Assignments } \\
\hline & & H1/H8 & $\mathrm{H} 2 / \mathrm{H} 7$ & $\mathrm{H} 4 / \mathrm{l}$ & & H9 & & \\
\hline & & 6.08 & 6.62 & 5.9 & & 7.62 & & \\
\hline
\end{tabular}

All assignments are for the proflavine-RNA complex at $282 \mathrm{~K}$. Values given in parentheses for ${ }^{31} \mathrm{P}$ assignments are for the unintercalated duplex. 

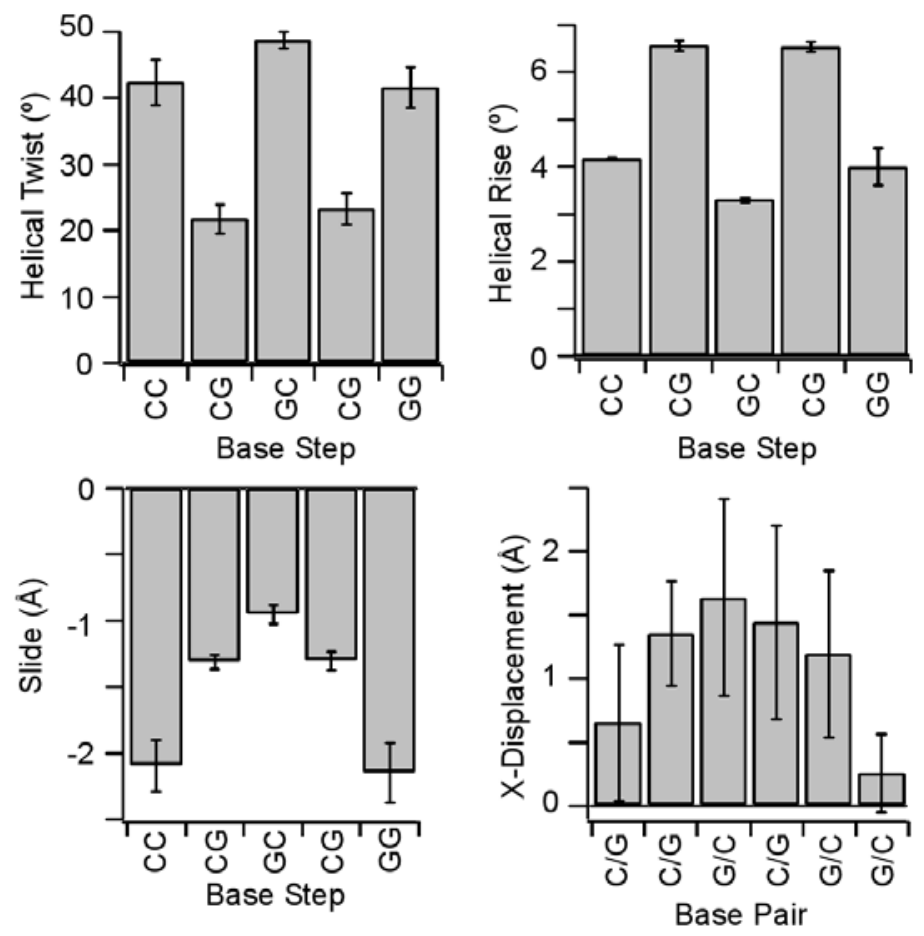

Figure S1. Bar graphs of average helical parameters for the 10 lowest energy structures of the $2^{\prime}, 5^{\prime}$ RNA duplex (GCCGCGGC) $)_{2}$ with proflavine bound at the two $\mathrm{CpG}$ steps. Only the central five out of the seven base steps are shown. Error bars indicate standard deviations. 

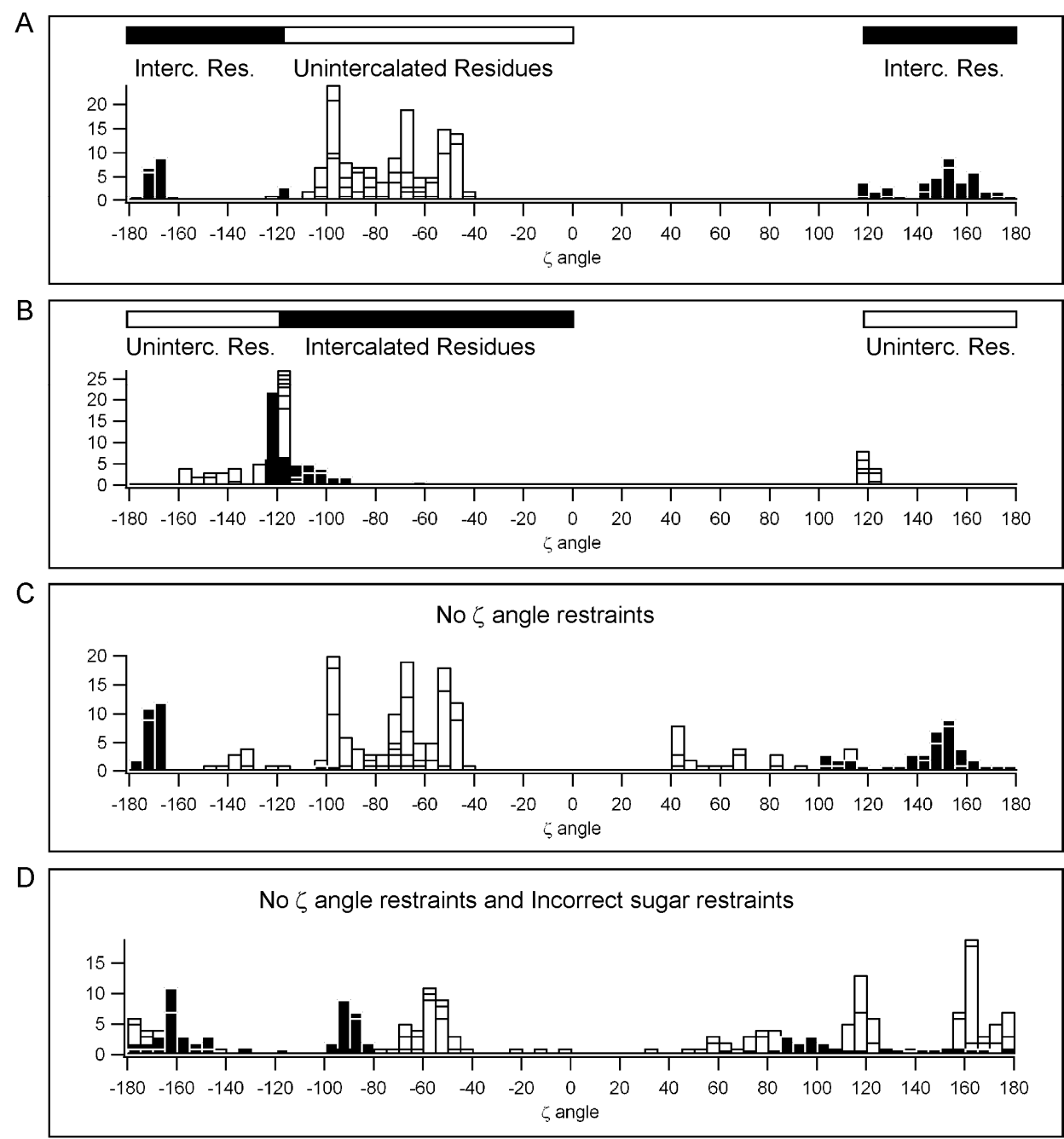

Figure S2. Zeta angles compiled from the 30 lowest energy structures after the second annealing refinement. Intercalated residue restraints correspond to $\zeta$ angles on the $\mathrm{C} 3 \mathrm{pG} 4$ and $\mathrm{C} 5 \mathrm{pG} 6$ phosphates. Unintercalated residue restraints correspond to all other $\zeta$ angles. A) $\zeta$ angles defined by what has been previously observed for $3^{\prime}, 5^{\prime}$-linked nucleic acids. B) $\zeta$ angles opposite of what was previously observed for $3^{\prime}, 5^{\prime}$-linked nucleic acids. C) No $\zeta$ angle restraints. D) No $\zeta$ angle restraints and the opposite sugar pucker restraints with respect to what is observed by NMR spectroscopy. 


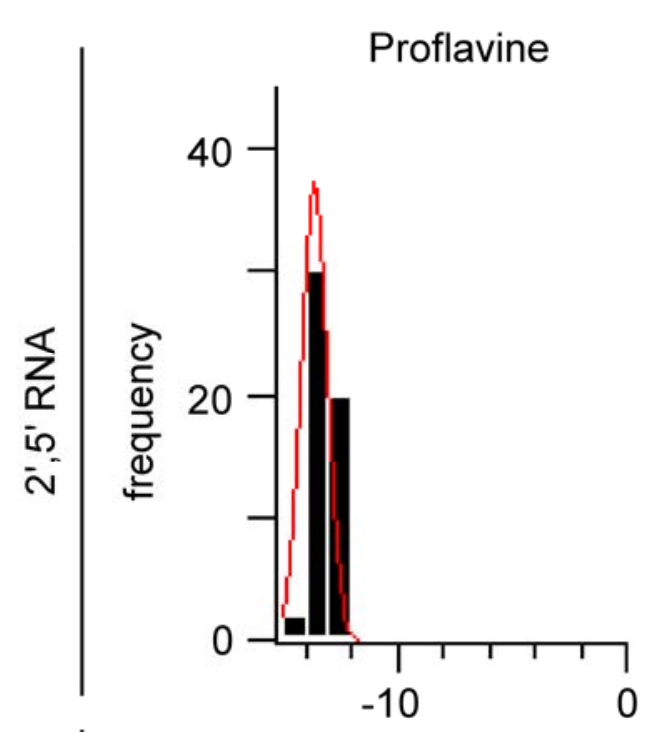

Acridine Orange

Ethidium
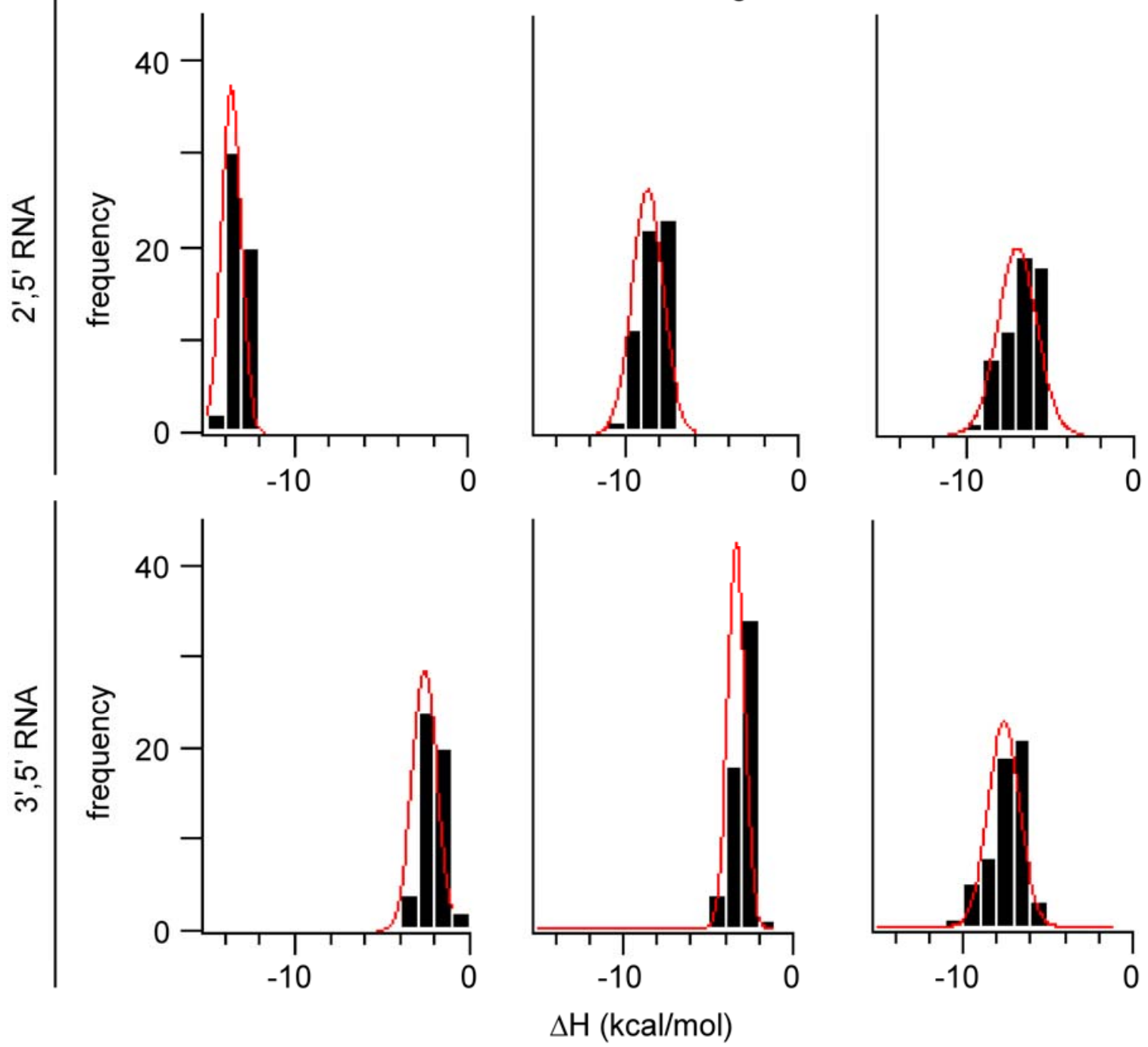

Figure S3. Histograms showing the enthalpy distributions obtained from model-free ITC.

\begin{tabular}{r|cc|cccc|}
\multicolumn{1}{c}{$\Delta \mathrm{G}_{2^{\prime}, 5^{\prime}}$} & $\Delta \mathrm{G}_{3^{\prime}, 5^{\prime}}$ & $\Delta \mathrm{H}_{2^{\prime}, 5^{\prime}}$ & $\Delta \mathrm{H}_{3^{\prime}, 5^{\prime}}$ & $-\mathrm{T} \Delta \mathrm{S}_{2^{\prime}, 5^{\prime}}$ & $-\mathrm{T} \Delta \mathrm{S}_{3^{\prime}, 5^{\prime}}$ \\
\cline { 2 - 7 } Proflavine & -7.8 & -5.9 & -13.6 & -2.6 & +5.8 & -3.3 \\
Acridine orange & -6.4 & -5.3 & -8.7 & -3.4 & +2.3 & -1.9 \\
Ethidium & -6.0 & -6.4 & -6.9 & -7.6 & +0.9 & +1.2 \\
\cline { 2 - 7 } & & & & &
\end{tabular}

Figure S4. Enthalpy $(\Delta \mathrm{H})$ and entropy $(-\mathrm{T} \Delta \mathrm{S})$ values for the intercalation of $2^{\prime}, 5^{\prime} \mathrm{RNA}$ and $3^{\prime}, 5^{\prime}$ RNA with proflavine, acridine orange, and ethidium at $25^{\circ} \mathrm{C}$. Data shown are in $\mathrm{kcal} / \mathrm{mol}$. 


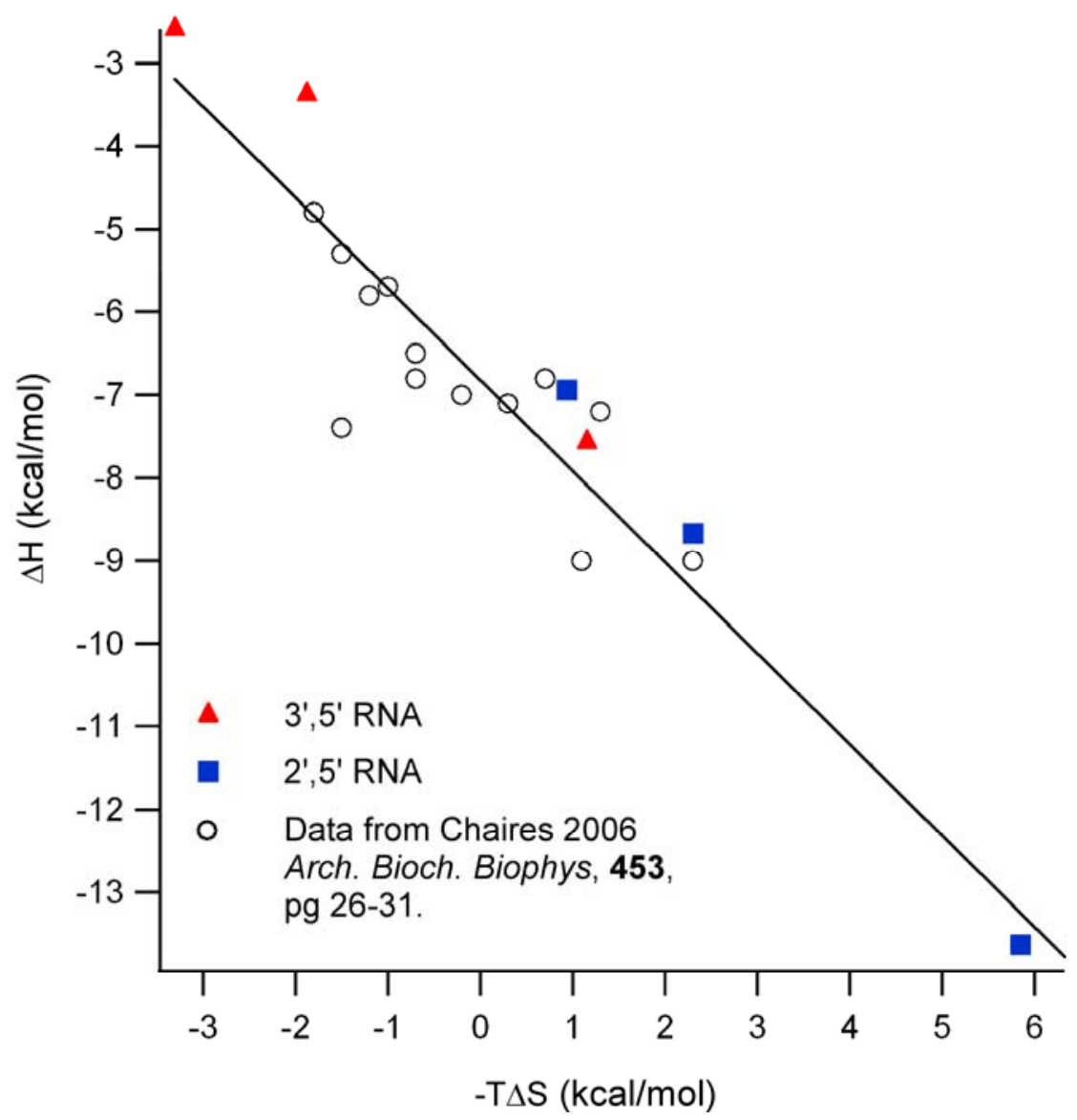

Figure S5. The linear relationship between enthalpy and entropy for intercalators binding to nucleic acids. The compilation of data reproduced from the work of Chaires ${ }^{1}$ shows the linear relationship for intercalators binding to DNA. The same trend is observed for $3^{\prime}, 5^{\prime}$ RNA as well as $2^{\prime}, 5^{\prime}$ RNA.

(1) Chaires, J. B., A thermodynamic signature for drug-DNA binding mode. Arch. Biochem. Biophys. 2006, 453, 26-31. 